\title{
Trends in Leprosy in the Kingdom of Bhutan, 1982-1992
}

\author{
P. JAKEMAN, * N. R. P. JAKEMAN \& J. SINGAY \\ National Leprosy Control Programme of Bhutan, Directorate of \\ Health Services, PO Box 100, Thimphu, Bhutan
}

\section{Accepted for publication 19 August 1994}

\begin{abstract}
Summary An evaluation programme was undertaken 11 years after the introduction of multidrug therapy (MDT) into Bhutan, by examining the case notes of 3239 leprosy patients who had been under treatment at any time during the period. The registered prevalence was found to have fallen markedly, as expected, and this had been accompanied by a clear fall in the case detection rate as well. The lepromatous rate among new patients rose considerably, giving epidemiological hope that the disease may be coming under control. However, no concomitant fall in the proportion of child cases was seen. The disability rate at detection rose slightly, although numbers were small. New cases were increasingly likely to have more highly positive skin smears, and to be selfreported. Programme planners should give thought to the implications of these findings.
\end{abstract}

\section{Introduction}

The small Himalayan Kingdom of Bhutan was among the first countries in the world to commit itself to offering MDT to all leprosy patients who needed it. The National Leprosy Control Programme had been established in 1981. This brought within a unified structure the existing leprosy control activities of two nongovernmental organizations, The Leprosy Mission and the Norwegian Santal Mission. It also established a single control area to be run by the Royal Government of Bhutan's Health Services.

MDT was begun in 1982, using the World Health Organisation (WHO) regime, ${ }^{1}$ but continuing the existing domiciliary strategy that was required by the remote, scattered population in the country. MDT was introduced gradually by each leprosy centre, covering hyperendemic pockets first in order to gain experience, and moving later into the areas where patients were more difficult to reach. Loose drugs were originally used,

* Corresponding author and present address: P. Jakeman, The Leprosy Mission Evaluation Unit, Katong PO Box 149, Singapore 9143 
individually counted as monthly calendar courses by the paramedical staff; more recently blister-calendar packs have been adopted, and some of the strict requirements for monthly face-to-face encounters have been relaxed to allow MDT coverage of patients living in very inaccessible areas.

In 1993, the requirement to evaluate the MDT programme arose, as planning was needed for the integration of leprosy services into primary health care. The likely epidemiological developments had to be predicted in order to estimate the future case load and staffing requirements.

\section{Method}

Central records were not found to be accurate for various reasons, and the registers of some programmes were not complete. In general, the case notes give a more complete record of patients' treatment, so 2 of the authors undertook a review of all available leprosy case notes in the country, covering the period 1982-92. In 6 centres, 3239 patients were identified. Data were extracted from the case notes manually, using a specially designed questionnaire, and were analysed using the Epi-Info Program. ${ }^{2}$ It is estimated that this study recovered the case notes of at least $85 \%$ of the actually registered patients, with a higher proportion from the most recent years.

The official population figure for Bhutan (1.4 million in 1993) is based on the annual updating of an estimate made many years ago, and is widely believed to be inaccurate. All calculations of rates in this study have therefore been based on the currrent informal estimate of 600,000 . Population growth in recent years has similarly been estimated to be $2 \%$ per annum, but has been at least partly balanced by large-scale emigrations of people who were originally counted as part of the population. Figures for child population are also not available. Prevalence figures were taken as cases registered at 31 December of each year of the study period. The programme is operational throughout the country, all detected cases are registered, and there is not thought to be a significant backlog of cases remaining to be detected.

Active case detection included mass survey at first, and all 18 districts have been surveyed at least once. Most districts were then resurveyed prior to the introduction of MDT, and some have had further surveys since. In recent years, fewer mass surveys of whole districts have been done, but they have been replaced by focal surveys of areas known to have been hyperendemic in the past. The frequency of these varies between 2 and 5 years. Other leprosy control activities (including other active casefinding methods - contact surveys and surveys of groups such as school children and servicemen) have remained unchanged throughout the study period. No alterations in case definition have occurred. Any patient who has ever had a positive skin smear has been regarded as multibacillary for the purposes of MDT. Multibacillary (MB) treatment is given for 24 doses, or thereafter until 3 consecutive negative skin smears are obtained, at least a month apart. Active post-MDT surveillance is maintained for 4 years after paucibacillary (PB) treatment, and for 10 years for MB cases. Disability is recorded by staff according to the previous WHO definition, ${ }^{3}$ and only visible deformity or damage (grades II-III) is included in this study. Patients with a slit-skin smear result of greater than $2+$ were defined as highly positive for the purposes of this study. 


\section{Results}

The complete data were analysed for trends in registered prevalence, MDT coverage and case detection rate (Figures 1 and 2). Additionally, the epidemiological characteristics of the 796 new cases detected during the study period were examined to assess whether the trends in these would give any indications on the course of the disease within the population, or on the operation of the programme (Table 1).

\section{REgistered PREVAlENCE, MDT COVERAge, AND CASE DETECTION RATE}

Registered prevalence was found to have fallen steadily throughout the study period, from 2534 to 143 patients. This is a fall from $42 \cdot 2$ per 10,000 to $2 \cdot 4$ per 10,000 . MDT coverage had risen to over $89 \%$ at the end of 1992 . The maximum number of patients taking MDT in any year was 861 in 1986, after which the actual numbers declined, although the coverage increased.

The case detection rate had fallen from 1.9 per 10,000 to 0.65 per 10,000 during the study period, that is from 114 to 39 new patients annually.

\section{NEW CASE CHARACTERISTICS}

The lepromatous rate was found to be gradually rising, from around $50 \%$ to around $70 \%$ by the end of the study period. The child rate varied between $3.7 \%$ and $12 \cdot 8 \%$, with a mean of $7 \cdot 1 \%$, but the numbers are small and the trend is unclear. The $\mathrm{M}: \mathrm{F}$ sex ratio varied between $1 \cdot 4: 1$ and $3 \cdot 8: 1$, but there was no clear trend away from the mean of $2 \cdot 4: 1($ male $=71 \%)$.

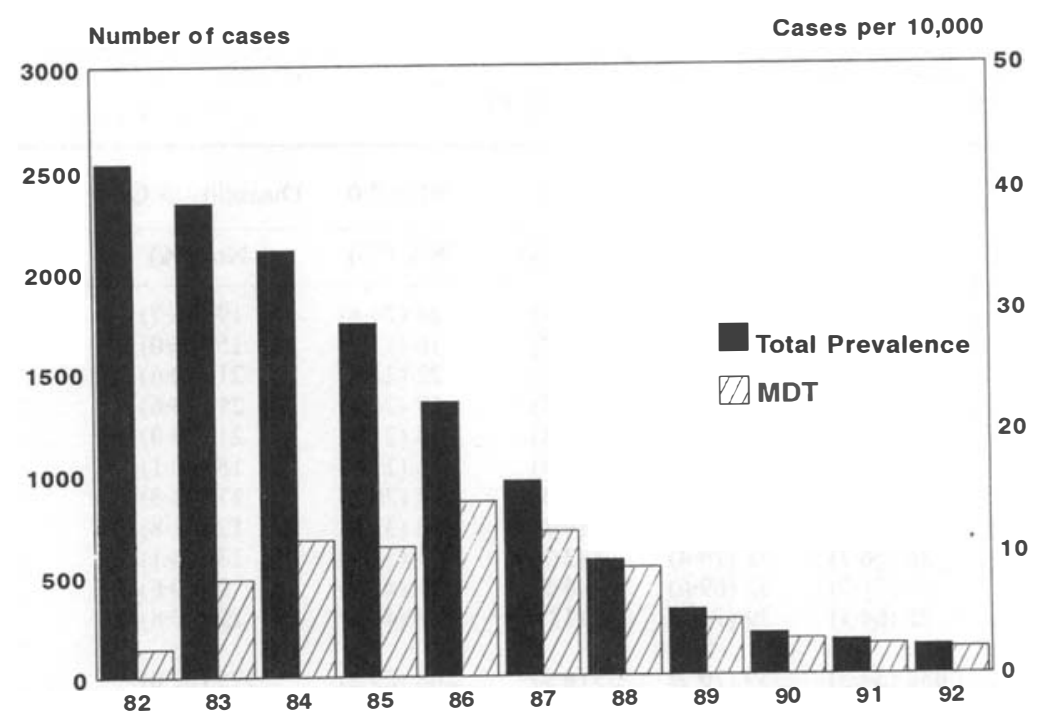

Figure 1. Prevalence of leprosy by treatment: MDT and total. 


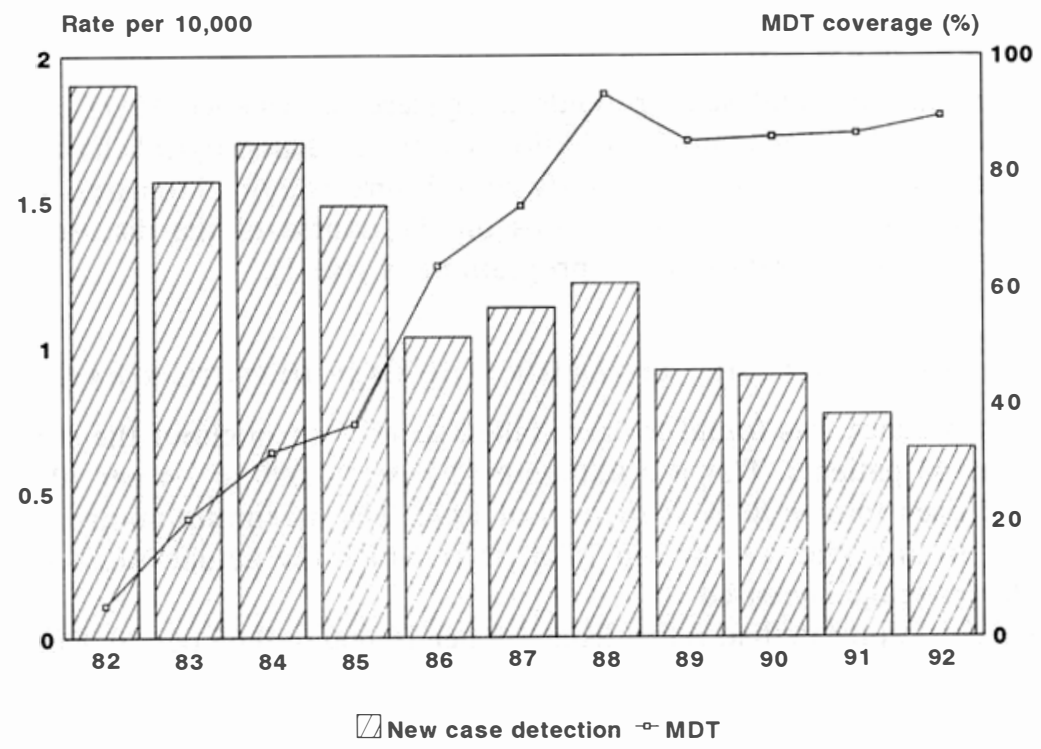

Figure 2. New case detection and MDT coverage.

\section{OPERATIONAL INDICATORS}

Certain indices give information that is useful in operational monitoring of the programme. The trend in disability rate shows a slight but definite increase, from under $20 \%$ to over $25 \%$, though the actual numbers fell . The mode of detection was not fully reported until recently, and so in only $491(62 \%)$ of the new cases in our study were these data available for analysis. However, a steadily increasing trend in the proportion

Table 1. Characteristics of new patients detected 1982-92

\begin{tabular}{|c|c|c|c|c|c|c|c|}
\hline & & MB & Male & Child & $\mathrm{BI}>2 \cdot 0$ & Disability > Gr 1 & Self-reported \\
\hline Year & Total & No. $(\%)$ & No. $(\%)$ & No. $(\%)$ & No. (\%) & No. $(\%)$ & No. $(\%)^{*}$ \\
\hline 1982 & 114 & $58(50 \cdot 9)$ & $67(58 \cdot 8)$ & $10(8 \cdot 8)$ & $28(24 \cdot 6)$ & $19(16 \cdot 7)$ & $10(14 \cdot 1)$ \\
\hline 1983 & 94 & $53(56 \cdot 4)$ & $63(67 \cdot 0)$ & $4(4 \cdot 3)$ & $16(17 \cdot 2)$ & $15(16 \cdot 0)$ & $16(37 \cdot 2)$ \\
\hline 1984 & 102 & $41(40 \cdot 2)$ & $79(77 \cdot 5)$ & $8(7 \cdot 8)$ & $22(21 \cdot 6)$ & $21(20 \cdot 6)$ & $12(17 \cdot 9)$ \\
\hline 1985 & 89 & $37(41 \cdot 6)$ & $58(65 \cdot 2)$ & $7(7 \cdot 9)$ & $19(21 \cdot 8)$ & $21(23 \cdot 6)$ & $8(12 \cdot 1)$ \\
\hline 1986 & 62 & $39(62.9)$ & $49(79 \cdot 0)$ & $3(4 \cdot 8)$ & $13(21 \cdot 7)$ & $21(33.9)$ & $11(29 \cdot 7)$ \\
\hline 1987 & 68 & $37(54.4)$ & $52(76 \cdot 5)$ & $4(5 \cdot 9)$ & $16(23.9)$ & $15(22 \cdot 1)$ & $15(34.9)$ \\
\hline 1988 & 73 & $35(47 \cdot 9)$ & $52(71 \cdot 2)$ & $4(5 \cdot 5)$ & $14(20 \cdot 0)$ & $17(23 \cdot 3)$ & $6(17 \cdot 1)$ \\
\hline 1989 & 55 & $38(69 \cdot 1)$ & $40(72 \cdot 7)$ & $5(9 \cdot 1)$ & $18(33 \cdot 3)$ & $12(21 \cdot 8)$ & $7(21 \cdot 3)$ \\
\hline 1990 & 54 & $36(66 \cdot 7)$ & $38(70 \cdot 4)$ & $2(3 \cdot 7)$ & $21(38.9)$ & $13(24 \cdot 1)$ & $14(37 \cdot 8)$ \\
\hline 1991 & 46 & $33(71 \cdot 7)$ & $32(69 \cdot 6)$ & $3(6 \cdot 5)$ & $21(45 \cdot 7)$ & $9(19 \cdot 6)$ & $15(48 \cdot 4)$ \\
\hline 1992 & 39 & $25(64 \cdot 1)$ & $29(74 \cdot 4)$ & $5(12 \cdot 8)$ & $15(40 \cdot 5)$ & $12(30 \cdot 8)$ & $10(35 \cdot 7)$ \\
\hline Total & 796 & $432(54 \cdot 3)$ & $559(70 \cdot 2)$ & $55(6 \cdot 9)$ & $203(25 \cdot 5)$ & $175(22 \cdot 0)$ & $124(25 \cdot 2)$ \\
\hline
\end{tabular}

\footnotetext{
* Percentage of those whose mode of detection was recorded.
} 
of self-reported cases is seen, from under $20 \%$ to over $35 \%$. In $780(98 \%)$ of the 796 new patients, a skin smear had been taken at detection and the result recorded. The percentage of new patients showing high smear positivity at detection increased from around $20 \%$ to around $40 \%$.

Data on relapse was also collected during the study. Only 3 PB cases were given second courses of PB treatment after having been released from treatment, though others had initial courses extended, and others were reclassified as MB. There were 15 cases of MB leprosy who were restarted on treatment after release because of a diagnosis of relapse. However, no standardized definition of multibacillary relapse had been published, and MB relapses were based on slightly positive smear results found during active surveillance. A review of individual case notes detected no case of genuine postMDT relapse among these cases.

\section{Discussion}

The decline in registered prevalence can be attributed both to the register-cleaning that preceded MDT introduction, and to the shorter duration of MDT treatment than of monotherapy. The initial rate of $4 \cdot 2$ per 1000 is already much lower than rates reported in $1969,{ }^{4}$ which gave district prevalences of 13.4 per 1000 in (low-endemic) Paro, and 24.8 per 1000 in (high-endemic) Lhuntsi, and this drop clearly occurred before MDT was introduced.

The MDT coverage did not increase consistently. There were some small groups of patients persistently not taking MDT, due to refusal or inaccessibility. As the overall numbers fell, they had a disproportionate effect on the coverage percentage.

The decline in case detection rate is less marked than the fall in registered prevalence, but is more significant in terms of the progress of leprosy control. ${ }^{5}$ These rates cannot, of course, be regarded as exactly representing the actual incidence. However, the amalgamation of results from 6 different centres, with differing case finding activities, means that the trend can be assumed to be reliable.

The fall in case detection rate started from the beginning of the period of this study, long before the influence of MDT can have had an effect. This may reflect the fact that Bhutan had a well-established and fairly comprehensive vertical monotherapy programme in place for some years before MDT was introduced. Other considerations are the introduction of BCG immunization ${ }^{6}$ in the 1960 s, which had attained effectively $100 \%$ coverage by the time Universal Child Immunization was achieved in 1991. The rapid socioeconomic improvements seen in the last 20 years as development activities were established may also have made a contribution. Although the case detection rate appears to fall more consistently in the second half of the study period, there is no overall change in the rate of fall. This finding is in keeping with recent findings from Malawi. ${ }^{7}$

The trends in the characteristics of the new cases can give some information on the progress of a control programme. ${ }^{5}$ There were no alterations of case definition during the study period, and the increase in the lepromatous rate can therefore be taken to represent a genuine change. This provides encouraging evidence that control of the disease is actually occurring. The child rate would be expected to fall as transmission decreases, and this has not been seen. Apart from the small numbers, which make trend analysis unreliable, a possible reason for this is the cautious over-diagnosis of 
single-lesion disease, especially in school surveys. The lack of an accurate denominator of the child population further confounds the interpretation, though the consistently low numbers are themselves an encouragement.

The $\mathbf{M}: \mathbf{F}$ sex ratio is higher than is usually reported. In Bhutan, health facilities are fully utilized by women, and although female paramedical staff are not employed within the leprosy programme, the examination of female patients by men is not usually a problem in the local culture. This suggests that there may be a real biological difference in the incidence between the sexes, and that neither the fall in prevalence nor the decrease in case detection rate brings this ratio towards unity.

The absence of MB relapse following MDT is likely to be irrelevant. The earliest cases treated with MDT had mostly been in the monotherapy treatment programme for many years. If they still harboured viable bacilli at the start of MDT, bactericidal treatment of almost any duration is likely to have eradicated these. The duration of this study is probably too short to expect to see genuine relapse from active cases treated with MDT. The matter of relapse among DDS-treated patients released from treatment without receiving MDT was not addressed in this study.

Any deterioration in disability rate is a worrying situation for programme managers, as it indicates a delay in case detection. In Bhutan, the delay is probably due to the decrease in the mass surveys that are being done, the overall low prevalence making such surveys non-cost-effective. The numbers of patients involved is small, but it is a matter of operational concern. The reduction in mass surveys contributes to the increasing proportion of self-reported patients, and this trend will need to be encouraged in order to promote early detection and prevention of disability, as well as to treat possible infectious cases.

As well as the epidemiological implications linked with the rising lepromatous rate, the increase in the proportion of new cases with high smear positivity is a trend that has operational implications as well. These patients who begin their MDT with a high bacterial load have a greater likelihood of reactions; and there are implications for the duration (and therefore cost) of treatment if the until 'smear negative treatment' regime continues to be used.

In interpreting all these results, however, the influence of active case finding has to be considered. There has been a reduction in mass surveys in recent years, largely due to the reduction in the team of vertical staff in response to the falling prevalence. This reduction by itself could explain away most of the encouraging findings of this study. However, the trends that are seen were established in the early part of the study period, when mass surveys were still an important strategy. These continued on a regular basis until 1988 , and then were gradually replaced by more cost-effective focal surveys in areas of previously known high prevalence. Contact and group surveys have continued throughout.

\section{Conclusion}

Bhutan has seen a remarkable drop in the registered prevalence of leprosy over the past 11 years, and the WHO target of 1 per 10,000 might be achieved by the year 1995, about 5 years before the global goal. This situation was reached through a comprehensive monotherapy programme followed by intensive MDT, with coverage approaching $90 \%$ 
in 1992. Many of the older cases treated with MDT may have actually been cured by years of dapsone monotherapy. Doubt has been expressed about the effect of MDT on the incidence of leprosy, and there is no change in the rate of fall of the case detection rate that can be attributed to MDT. The time elapsed since adequate MDT coverage has been achieved may still be too short for an effect to be seen. The lepromatous rate, rising to $70 \%$, seems to confirm that the disease is coming under control, although the child rate has not fallen as would be expected. A small rise in the disability rate has been seen, but this may be reversed in time by encouraging early self-reporting by patients. The increasing number of patients who are now starting MDT with highly positive skin smears is important in planning the programme, both in ensuring staff awareness about lepra reactions, and in decision-making about programme policy and the economics of the MDT delivery options.

\section{Acknowledgments}

This study would have been impossible without the help and co-operation of numerous colleagues, both national and expatriate, and their contribution is warmly acknowledged, as is the permission of the Royal Government of Bhutan for publication. Some of the patients studied had received MDT in blister-calendar packs donated by CibaGeigy. Dr W. C. S. Smith made valuable suggestions on earlier versions of this paper, as did 2 anonymous referees. P.J. is employed by The Leprosy Mission International.

\section{References}

1 WHO Study Group. Chemotherapy of leprosy for control programmes. Technical Report Series No. 675. WHO: Geneva, 1982.

${ }^{2}$ Dean AG et al. Epi-Info, Ver 5: a word processing, data base and statistics program for epidemiology on microcomputers. Stone Mountain: USD inc, 1990.

3 WHO. Expert Committee on Leprosy: fourth report. Technical Report Series No. 459. WHO: Geneva, 1970.

${ }^{4}$ Berkeley JS. Leprosy in Bhutan: a pilot survey. Lepr Rev, 1972; 42: 188-96.

5 Noordeen SK. The epidemiology of leprosy. In: Hastings, RC (ed) Leprosy. Edinburgh: Churchill Livingstone, 1985.

6 Fine PEM. Reflections on the elimination of leprosy. Editorial, Int J Lepr, 1992; 60 (1): 71-80.

7 Boerrigter G, Pönninghaus J. Does the introduction of WHO-MDT influence trends in leprosy?-the Malaŵian experience. Lepr Rev, 1993; 64: 227-35. 